\title{
Ajuste de funciones de ahusamiento a los perfiles fustales de cinco Pináceas de la región de EI Salto, Durango
}

\author{
Sacramento Corral R. ${ }^{1}$ \\ José de Jesús Návar Chaidez ${ }^{1}$ \\ Fabián Fernández Sánchez ${ }^{2}$
}

\section{RESUMEN}

Los objetivos del presente trabajo fueron: 1) estimar parámetros y 2) validar 7 funciones de ahusamiento ajustadas a los perfiles fustales sin corteza de 5 especies comerciales de pino de la región de El Salto, Durango. Los diámetros sin corteza se midieron a diferentes alturas en árboles derribados en operaciones de aprovechamiento. El número total de árboles utilizado para cada especie fue de 507 de Pinus cooperi, 453 de $P$. durangensis, 424 de $P$. engelmannii, 400 de $P$. leiophylla y 322 de $P$. herrerae, de los cuales el $75 \%$ se utilizaron para estimar parámetros y el $25 \%$ para validar los modelos de Clutter (1980); Amidon (1984); Biging (1984); Kozak (1988); Kozak, Rustagi y Loveless (1991) y Newnham (1992). Los parámetros se ajustaron por medio de regresión lineal, intrínsecamente lineal, polinomial, múltiple y no lineal. La prueba de validación consistió en comparar los estadísticos; coeficiente de determinación, el error estándar y el sesgo, incluyendo la distribución, normalidad y varianza común de los errores. Los resultados mostraron que el modelo de Biging (1984) predice mejor el perfil diamétrico sin corteza de las cinco especies, porque definió mejor la tendencia de los perfiles diamétricos, mostró uno de los coeficientes de determinación más altos, errores estándares más bajos y errores distribuidos más normalmente. Por esta razón se recomienda este modelo en la futura estimación de los perfiles fustales de las pináceas del área de estudio. El modelo estima adecuadamente el volumen fustal para árboles con diámetros menores a 30 $\mathrm{cm}$ pero requiere de investigación adicional para estimar volúmenes comerciales de árboles con diámetros mayores a $30 \mathrm{~cm}$.

PALABRAS CLAVE:

Biging, El Salto, Durango, funciones de ahusamiento, México, pináceas.

\section{ABSTRACT}

The objectives of this work were: 1) to estimate parameters and 2) to validate 7 taper functions fitted to stem profiles of 5 commercial pine species of the El Salto, Durango region. Measurements of diameter at several stem heights were conducted in fallen trees during harvesting operations. The total number of trees measured for each species was: 507 (Pinus cooperi), 453 ( $P$. durangensis), 424 ( $P$. engelmannii), 400 ( $P$. leiophylla), and 322 ( $P$. herrerae) of which $75 \%$ were used to estimate parameters and the remaining $25 \%$ to test

1 Facultad de Ciencias Forestales, Universidad Autónoma de Nuevo León. Km. 145 Carretera Nacional Linares, N. L. 67700 México.jnavar@ccr.dsi.uanl.mx

2 Instituto Tecnológico Forestal No. 1. Mesa del Tecnológico s/n. Apdo. Postal No. 2, El Salto P. N., Durango. 34950. México.

Manuscrito recibido para su publicación el 14 de diciembre de 1998. 
the goodness of fit of taper fuctions of Clutter (1980), Amidon (1984), Biging (1984), Kozak (1988), Kozak y Newnham (1992). Parameters were fitted by least square techniques in linear, polinomial and multiple regression analysis. The goodness of fit test consisted of comparing the statistics, coefficient of determination, standard error, and skewness, as well as, normality, randomness, and common variance of errors. The results showed that the model of Biging (1984) estimated better the stem profiles of the five pine species since it showed one of the largest coefficient of determination, one of the smallest standard errors and errors were distributed close to the normal. For this reason, this model is recommended to estimate stem profiles of the pine species of the study area. The model estimates accurately stem volume for trees with diameter smaller than $30 \mathrm{~cm}$ but it requires additional research to estimate merchanteable volume of trees with diameters larger than $30 \mathrm{~cm}$.

KEY WORDS:

Biging, El Salto, Durango, taper functions, México, pine species.

\section{INTRODUCCION}

Los retos de los manejadores silvícolas son cada día mayores por las restricciones ambientales en los sistemas de planeación forestal a largo plazo. Uno de estos es la necesidad de técnicas precisas que describan los volúmenes y distribución de productos forestales derivados de árboles del género Pinus, como un principio del manejo forestal sustentable. La elaboración de planes de manejo e inventarios forestales basan su filosofía operativa en estos conceptos. Los modelos de ahusamiento, utilizados desde hace varias décadas, definen objetivamente el volumen y distribución de productos derivados de fustes de árboles.
Existen funciones de ahusamiento ajustadas a varias especies de coníferas del país. Torres et al. (1993) prueba varios modelos para ocho especies forestales del estado de México. Rentería (1995) estima el volumen comercial de Pinus cooperi mediante modelos de ahusamiento en el estado de Durango. Návar et al. (1997), prueba siete funciones de ahusamiento para Pinus hartwgii en el Noreste de México. Zepeda et al. (1997), prueba varias funciones para las especies de Pinus arizonica, Pinus durangensis y Pinus engelmannii en la Sierra Madre Occidental del estado de Chihuahua. Sin embargo para las especies comerciales del estado de Durango, pocos trabajos han descrito matemáticamente el ahusamiento.

\section{OBJETIVOS}

Los objetivos de este trabajo fueron: 1) estimar parámetros y 2) validar siete funciones de ahusamiento ajustadas a cinco especies comerciales de pino en la región del Salto Durango, México.

\section{MATERIALES Y METODOS}

Descripción general del área de estudio.

El estudio se realizó en la región forestal de El Salto, Durango, la cual comprende los predios de la Unidad de Conservación y Desarrollo Forestal No. 6, localizándose en el sistema montañoso denominado Sierra Madre Occidental. Esta pertenece a las subprovincias Gran Meseta, cañones duranguenses y mesetas $y$ cañones del sur, se encuentra entre las coordenadas $23^{\circ} 30^{\prime}$ a $24^{\circ} 15^{\prime}$ de latitud Norte y $105^{\circ} 15^{\prime}$ a $105^{\circ} 45^{\prime}$ de longitud Oeste, a $100 \mathrm{Km}$, al suroeste de la ciudad de Durango. Las alturas sobre el nivel del mar fluctúan entre 1,400 y 2,600 metros. La región se encuentra dentro del grupo de climas templados $C$, sub-grupo de climas semicálidos $(A) C\left(W_{1}\right)$ y tipos semicálidos sub-húmedos con lluvias en verano con un 
porcentaje de lluvia invernal entre 5 y 10.2 $\mathrm{mm}$, con precipitación media anual de 800 $1200 \mathrm{~mm}$ y una temperatura media anual de $20-22^{\circ} \mathrm{C}$. La geología de la región consiste en rocas ígneas extrusivas ácidas y basaltos del cretásico superior y cretásico inferior. Las rocas son del tipo sedimentario y vulcanoso sedimentario. Los tipos de suelos encontrados son Litosol, Cambisol, Regosol y Feozem.

Por su ubicación geográfica, la zona presenta diversas condiciones de vegetación que va desde selva baja caducifolia, masas puras de encino y pino y bosque mezclados de pino-encino. Las especies de mayor valor comercial, tanto por las características tecnológicas de su madera como por su rango de distribución son las del género pino, con las especies listadas en orden de importancia por su volumen de aprovechamiento: $P$. cooperi Blanco, $P$. durangensis Martínez, $P$. leiophylla Schl et Cham, $P$. engelmannii Carr, $P$. cooperi var ornelasi, $P$. teocote et Cham, $P$. herrerae Martínez. Otras especies de menor valor comercial, distribución y abundancia son $P$. ayacahuite Ehrenb, $P$. lumholtzii Rob et Fern, $P$. douglasiana Martínez, $P$. michoacana cornuta Martínez, $P$. oocarpa Schiede. Además se aprovechan algunas especies de encino (Quercus spp) y otras especies asociadas con las coníferas y hojosas de los géneros Arbutus, Juniperus, Pseudotsuga, Abies y Picea.

\section{METODOLOGIA}

Las mediciones de diámetro sin corteza - altura se realizaron directamente de árboles derribados en operaciones de aprovechamiento, distribuidos en toda el área de estudio tratando de cubrir todas las condiciones de sitio presentes y todas las categorías de diámetro y altura. El tamaño de muestra por especie fue de 507 árboles de $P$. cooperi, 453 de $P$. durangensis, 424 de $P$. engelmannii, 400 de $P$. leiophylla y 322 para $P$. herrerae, de los cuales el $75 \%$ de cada especie se seleccionó y separó aleatoriamente para el ajuste estadístico de parámetros de los modelos y el $25 \%$ restante para la validación de los modelos. El diámetro sin corteza se midió a la base del fuste a $0.30 \mathrm{~m}$, a $1.30 \mathrm{~m}$ y desde aquí hasta la parte distal a cada metro. Otras variables medidas fueron: diámetro normal, edad, altura del tocón, grosor de corteza y altura total.

\section{Modelos ajustados.}

La nomenclatura utilizada en este trabajo es la siguiente:

$\mathrm{D}=$ Diámetro normal a $1.30 \mathrm{~m}$ de altura (m).

$\mathrm{H}=$ Altura total del fuste $(\mathrm{m}) ; \mathrm{H}_{\mathrm{a}}=\mathrm{H}-$ $1.37(\mathrm{~m})$.

$\mathrm{P}=\mathrm{H}_{\mathrm{l}} / \mathrm{H}$ (sin dimensiones).

$\mathrm{Z}=\mathrm{h} / \mathrm{H}$ (sin dimensiones).

$X=(\mathrm{H}-\mathrm{h}) /(\mathrm{H}-1.30 \mathrm{~m})$ (sin dimensiones).

$D_{1}=$ Diámetro al punto de inflexión de la forma neiloide a la forma cuadrática del fuste, tomado como el $\mathrm{d}^{\mathrm{a}} \mathrm{h}_{.067 \mathrm{D}}$ (m).

$\mathrm{H}_{1} \quad$ = Altura del fuste al punto de inflexión descrito $(\mathrm{m})$.

$X_{k}=\left(1-Z^{1 / 2}\right) /\left(1-P^{1 / 2}\right)$ (sin dimensiones).

$\mathrm{h}=$ Altura del fuste sobre el tocón (m).

$\mathrm{d}=$ Diámetro sin corteza del fuste a la altura $h(m)$.

$B_{0}, B_{1}, B_{2}, B_{3}, \quad B_{4}, \quad c=$ Parámetros estadísticos.

Los modelos de ahusamiento utilizados para describir el perfil del fuste de las cinco especies fueron los siguientes:

1. El modelo de Clutter (1980), dado por la ecuación:

$$
d=\beta_{1}^{*}\left(D^{\beta 2}\right) *\left(H^{\beta 3}\right) *(H-h)^{\beta 4}
$$

2. El modelo de Amidon (1984), dado por la ecuación:

$$
d=\beta_{1} \frac{D(H-h)}{(h-1.30)}+\beta_{2} \frac{\left(H^{2}-h^{2}\right)(h-1.30)}{H^{2}}
$$


3. El modelo de Biging (1984), dado por la ecuación:

$$
d=D\left[\beta 1+\beta 2 \ln \left(1-\left(1-\exp \left(-\frac{\beta 1}{\beta 2}\right)\right)\left(\frac{h}{H}\right)^{1 / 3}\right)\right]
$$

4. El modelo de Kozak (1988) fue trabajado estadísticamente en dos formas; la primera (Kozak1), está representada por la ecuación:

$$
\frac{d}{D_{1}}=\left[\frac{1-\sqrt{Z}}{1-\sqrt{P}}\right]^{c}
$$

5. La segunda forma (Kozak2) está representada como sigue:

$$
\ln (d)=f\left(\ln \left(X_{k}\right), Z, \ln (Z), \exp ^{Z}, \sqrt{Z}, \frac{D}{H}\right)
$$

6. El modelo modificado de la forma variable presentado por Newnham (1992), esta representado por la ecuación:

$$
\ln \left(\frac{d}{D}\right)=f\left(X, \ln (X), \frac{D}{H}, \frac{\frac{D}{H}}{\sqrt{h}}, \frac{H}{\sqrt{h}}\right)
$$

7. El modelo de Rustagi y Loveless (1991) está dado por la ecuación:

$$
d=\left(\beta_{0}+\beta_{1}{ }^{*} D\right)\left[\frac{H-h}{H_{a}}\right]^{\beta_{2}}
$$

\section{Procedimiento Estadístico.}

En el ajuste de las funciones de ahusamiento se utilizaron los siguientes pares datos de diámetro sin corteza y altura por especie, 3,624 de $P$. cooperi, 3,140 de $P$. durangensis, 2,703 de $P$. engelmannii, 2,527 de $P$. leiophylla y 2,184 de $P$. herrerae, de los cuales se estimaron los parámetros estadísticos de las siete ecuaciones de ahusamiento. Para el ajuste se utilizó la técnica de cuadrados mínimos en regresión múltiple, intrínsecamente lineal, polinomial y no lineal (sin derivadas parciales, DUD) del paquete estadístico SAS. Para las ecuaciones que necesitaron transformaciones, los parámetros estadísticos se calcularon con las transformaciones necesarias para estimar el verdadero valor del $r^{2}$ y el error estándar estimado (SEE) en sus dimensiones originales.

La prueba de validación se realizó con datos de diámetro sin corteza - altura del 25 $\%$ de la muestra total correspondiente a los pares de datos de 1,241 para $P$. cooperi, 1,046 para $P$. durangensis, 952 para $P$. engelmannii, 963 para $P$. leiophylla y 768 para $P$. herrerae. Los estadísticos de comparación entre los modelos y entre las especies fueron el sesgo promedio, $r^{2}$, SEE, y bondad de predicción del perfil diamétrico.

Estos estadísticos se estimaron como sigue:

$$
\text { Sesgo Promedio }=\frac{\sum_{i=1}^{\mathrm{n}}(\mathrm{Yi}-\hat{\mathrm{Y} i})}{\mathrm{n}}
$$

$$
\begin{aligned}
& r^{2}=1-\frac{\sum_{i=1}^{n}\left(Y_{i}-\widehat{Y}_{i}\right)^{2}}{\sum_{i=1}^{n}\left(Y_{i}-\bar{Y}\right)^{2}} \\
& \text { SEE }=\left[\frac{\sum_{i=1}^{n}\left(Y_{i}-\bar{Y}_{i}\right)^{2}}{n-p}\right]^{\frac{1}{2}}
\end{aligned}
$$


donde: $Y_{i}$ es el valor observado o la variable dependiente, $\bar{Y}$ es el promedio de los datos observados, $\bar{Y}$ es el valor predicho y $p$ es el número de parámetros del modelo, incluyendo la intercepta.

También se realizó el análisis de los errores para observar su distribución, aleatoriedad y varianza común y tener una mayor certidumbre en la selección del mejor modelo. Otras distribuciones consideradas fueron: la kurtosis, sesgo, y la probabilidad de normalidad de los errores. La determinación del mejor modelo resultó de la ponderación de los diferentes parámetros, los cuales se calificaron por orden de importancia como sigue: $\mathrm{r}^{2}$, SEE y sesgo promedio del 75 y $25 \%$ de los datos, así como kurtosis, sesgo, la probabilidad de normalidad de los errores, el número de coeficientes de las regresiones con su valor de probabilidad y finalmente la bondad de predicción del perfil diamétrico.

\section{Características de los árboles muestra.}

Los estadísticos de los parámetros dasométricos de los árboles, separados en 75 y $25 \%$ respectivamente se presentan en la Tabla 1, los cuales no presentaron diferencias significativas entre las especies. La variación indica que la muestra esta distribuida en todas las categorías de edad, altura y diámetro.

\section{RESULTADOS Y DISCUSION}

Los estadísticos del ajuste de los siete modelos mostraron diferencias significativas entre las cinco especies (Tabla 2). Los valores promedios del coeficiente de determinación ajustado por el número de parámetros indicaron que el modelo de Amidon presenta el mejor ajuste (0.982), seguido por los modelos de Newnham (0.963), Biging (0.962) y Rustagi y Loveless (0.956), finalmente los que mostraron ajustes menos bondadosos fueron los modelos de Kozak1 (0.948), Clutter (0.928) y Kozak2 (0.913) (Tabla 2).
Los errores estándar promedio para las especies indicaron que los modelos de Newnham (2.084), Biging (2.140) y Rustagi y Loveless (2.197) fueron los mejores y los modelos de Amidon (2.287), Kozak1 (2.364), Clutter (2.813) y Kozak2 (3.083) mostraron las variaciones mayores. Los coeficientes de sesgo promedio de los modelos de Clutter (-0.046), Rustagi y Loveless $(-0.072)$ y Amidon $(-0.094)$ presentaron los valores más cercanos a cero y los modelos de Newnham (-0.126), Kozak1 (0.144), Biging (-0.164) y Kozak2 $(-1.330)$ tuvieron las desviaciones de cero más notorias (Tabla 2).

Los valores de kurtosis promedio más aproximados al valor de la distribución normal lo presentaron los modelos de Amidon (3.34), de Rustagi y Loveless (2.65), de Kozak2 (2.55), de Clutter (2.49) y de Biging (2.23). Los modelos con valores de Kurtosis mas alejados de la normalidad fueron Kozak1 (5.36) y Newnham (23.11). Los modelos con el sesgo promedio de los errores con valores más cercanos a cero fueron Rustagi y Loveless (0.19), Kozak2 $(-0.23)$, Biging (0.24) y Newnham $(-0.35)$, mientras que los sesgos promedio con mayores desviaciones del cero lo presentan los modelos de Clutter (-0.40), Amidon (0.58) y Kozak1 (-0.96). La prueba de la normalidad de los errores resultó aceptar la hipótesis alterna de otra distribución, para todos los modelos ( $p>w=0.01)$.

Los modelos que presentaron valores de ponderación mejores y en forma consistente fueron Biging, Amidon, Newnham y Rustagi y Loveless. El primer modelo sólo presentó uno de los mayores sesgos promedio $(-0.164)$. El segundo modelo presentó uno de los mayores valores de SEE porque la ecuación describe una curva cuadrática, como fue también observado por Návar et al. (1997) para $P$. hartwegii, la cual no tiende a simular la curva sigmoide característica del perfil fustal. El modelo de Newnham presentó debilidades en los estadísticos de kurtosis, concentrando las desviaciones en valores 
cercanos a cero. Finalmente el modelo de Rustagi y Loveless no explicó el ahusamiento en todas las categorías de diámetro puesto que tiende a formar también una curva cuadrática que no converge en un punto de inflexión en los árboles de mayores dimensiones.

Los resultados de validación indicaron que los valores promedio de los coeficientes de determinación descendieron ordenadamente en los modelos de Biging (0.955), Amidon (0.952), Newnham (0.948), Rustagi y Loveless (0.948), Kozak1 (0.930),
Clutter (0.928) y Kozak2 (0.906) (Tabla 3). Los valores promedio de los errores ordenados ascendentemente fueron para los modelos de Amidon (2.064), Newnham (2.076), Biging (2.078), Rustagi y Loveless (2.128), Kozak1 (2.412), Clutter (2.482) y Kozak2 (2.803). El sesgo promedio ordenado crecientemente se comporta de la siguiente manera para los modelos de: Amidon (-0.030), Biging (0.074), Clutter (0.258), Rustagi y Loveless (0.310), Newnham (0.402), Kozak1 (0.490) y Kozak2 $(-0.053)$ (Tabla 3).

Tabla 1. Estadísticos de 5 especies de pino de la región del Salto, Durango, México

\begin{tabular}{|c|c|c|c|c|c|}
\hline \multirow[b]{2}{*}{ ESPECIE } & \multicolumn{3}{|c|}{ AJUSTE } & \multicolumn{2}{|c|}{ VALIDACION } \\
\hline & Estadísticos & $\begin{array}{l}\text { Diámetro } \\
(\mathrm{cm})\end{array}$ & $\begin{array}{l}\text { Altura } \\
(\mathrm{m})\end{array}$ & $\begin{array}{l}\text { Diámetro } \\
(\mathrm{cm})\end{array}$ & $\begin{array}{l}\text { Altura } \\
(\mathrm{m})\end{array}$ \\
\hline P. cooperi & $\begin{array}{l}\text { Promedio } \\
\text { DS } \\
\text { Rangos }\end{array}$ & $\begin{array}{c}26.6 \\
10.9 \\
(7.6-66.5)\end{array}$ & $\begin{array}{c}17.3 \\
6.1 \\
(2.3-34.0)\end{array}$ & $\begin{array}{c}24.3 \\
10.3 \\
(8.1-58.9)\end{array}$ & $\begin{array}{c}16.8 \\
6.0 \\
(3.1-33.2)\end{array}$ \\
\hline P. durangensis & $\begin{array}{l}\text { Promedio } \\
\text { DS } \\
\text { Rangos }\end{array}$ & $\begin{array}{l}24.3 \\
10.0 \\
(7.5-54.8)\end{array}$ & $\begin{array}{c}16.4 \\
6.4 \\
(2.2-35.0)\end{array}$ & $\begin{array}{c}20.1 \\
8.6 \\
(7.6-44.2)\end{array}$ & $\begin{array}{c}15.3 \\
6.3 \\
(2.4-29.4)\end{array}$ \\
\hline P. engelmannii & $\begin{array}{l}\text { Promedio } \\
\text { DS } \\
\text { Rangos }\end{array}$ & $\begin{array}{c}21.7 \\
9.6 \\
(7.6-55.0)\end{array}$ & $\begin{array}{c}13.9 \\
5.6 \\
(2.2-30.1)\end{array}$ & $\begin{array}{c}19.3 \\
8.6 \\
(8.1-50.0)\end{array}$ & $\begin{array}{c}12.6 \\
4.1 \\
(2.1-22.5)\end{array}$ \\
\hline P. leiophylla & $\begin{array}{l}\text { Promedio } \\
\text { DS } \\
\text { Rangos }\end{array}$ & $\begin{array}{c}21.5 \\
9.7 \\
(7.5-50.4)\end{array}$ & $\begin{array}{c}13.4 \\
5.5 \\
(2.5-27.0)\end{array}$ & $\begin{array}{c}20.5 \\
7.6 \\
(7.8-40.1)\end{array}$ & $\begin{array}{c}13.8 \\
4.9 \\
(3.5-26.0)\end{array}$ \\
\hline P. herrerae & $\begin{array}{l}\text { Promedio } \\
\text { DS } \\
\text { Rangos }\end{array}$ & $\begin{array}{c}21.3 \\
9.6 \\
(8.1-52.8)\end{array}$ & $\begin{array}{c}13.5 \\
5.5 \\
(3.0-27.6)\end{array}$ & $\begin{array}{c}23.7 \\
9.6 \\
(8.1-49.5)\end{array}$ & $\begin{array}{c}15.9 \\
5.5 \\
(3.8-26.6)\end{array}$ \\
\hline
\end{tabular}

DS= Desviación estándar, Rangos (Mínimo - Máximo). 
Tabla 2. Estadísticos promedio del ajuste de parámetros de 7 funciones de ahusamiento a los perfiles fustales sin corteza de 5 especies pino de la región del Salto, Durango, México

\begin{tabular}{|c|c|c|c|c|c|c|c||}
\hline \multirow{2}{*}{ MODELO } & \multicolumn{4}{|c|}{ ESTADISTICOS DEL MODELO } & \multicolumn{3}{c||}{ ESTADISTICOS DE LOS } \\
\hline$\left(\mathrm{M}_{\mathrm{j}}\right)$ & $\mathrm{R}^{2}$ & SEE & C.V & Sesgo & Kur & Sesgo & P $>\mathrm{W}$ \\
\hline $\mathrm{C}$ & 928 & 2813 & 20710 & -46 & 2490 & -400 & 1 \\
\hline $\mathrm{A}$ & 982 & 2287 & 16974 & -94 & 3340 & 584 & 1 \\
\hline $\mathrm{B}$ & 962 & 2140 & 15864 & -164 & 2238 & 246 & 1 \\
\hline $\mathrm{KI}$ & 948 & 2365 & 17498 & 144 & 5360 & -964 & 8 \\
\hline $\mathrm{K} 2$ & 913 & 3083 & 22734 & $-1-330$ & 2552 & -234 & 1 \\
\hline $\mathrm{N}$ & 963 & 2085 & 13022 & -126 & 23112 & -354 & 1 \\
\hline $\mathrm{R}$ & 956 & 2197 & 16290 & -72 & 2654 & 190 & 1 \\
\hline
\end{tabular}

Nota: Modelos (C = M. Clutter, A =. Amidon, B = M. Biging, K1 = M. Kozak1, K2 = M. Kozak2, N = M. Newnham, $\mathrm{R}=\mathrm{M}$. Rustagi).

Tabla 3. Estadísticos promedio de la validación de 7 funciones de ahusamiento a los perfiles fustales sin corteza de 5 especies pino de la región del Salto, Durango, México

\begin{tabular}{||c|c|c|c|c|c|c|c||}
\hline \hline \multirow{2}{*}{ MODELO } & \multicolumn{4}{|c|}{ ESTADISTICOS DEL MODELO } & \multicolumn{3}{c||}{ ESTADISTICOS DE LOS } \\
\hline ERRORES \\
\hline $\mathrm{Mj})$ & $\mathrm{R} 2$ & SEE & C.V & Sesgo & Kur & Sesgo & P $>$ W \\
\hline C & 928 & 2482 & 19284 & $0-258$ & 199962 & -378 & 3 \\
\hline B & 952 & 2064 & 16266 & -30 & 3980 & 276 & 0 \\
\hline KI & 955 & 2078 & 15880 & $0-074$ & 4910 & -98 & 0 \\
\hline K2 & 930 & 2412 & 19314 & 490 & 10004 & -930 & 9 \\
\hline N & 948 & 2803 & 22374 & -530 & 6088 & -800 & 0 \\
\hline R & 948 & 2128 & 16886 & 310 & 4494 & -70 & 0 \\
\hline \hline
\end{tabular}

Nota: Modelos ( $=$ = M. Clutter, A = M. Amidon, B = M. Biging, K1 = M. Kozak1, K2 = M. Kozak2, N = M. Newnham, $\mathrm{R}=\mathrm{M}$. Rustagi). 
Los errores mostraron valores de kurtosis de 4.91, 3.98, 42.07 y 3.98 para los modelos de Biging, Amidon, Newnham y Rustagi y Loveless, respectivamente. El promedio del sesgo del error fue de -0.09 , $0.27,-1.76,-0.07$, para los cuatro modelos descritos anteriormente, respectivamente. Todos los modelos registraron valores alejados de la normalidad por concentrar la mayoría de los errores en el centro y poseer una kurtosis alta.

Los estadísticos ponderados de comparación considerados para los siete modelos indicaron que la función de Biging se ajustó mejor a los perfiles diamétricos de las cinco especies estudiadas. Este modelo tiende a describir adecuadamente el ahusamiento sigmoidal de los árboles para todas las categorías diamétricas. El resto de los modelos presentaron curvas cuadráticas sencillas o sigmoidales con direcciones caprichosas en categorías diamétricas extremas (Fig. 1). El modelo de Biging tiene la ventaja de que presenta dos parámetros estadísticos con una variable, en contraste con los modelos polinomiales usados por Rentería et al. (1995) y Torres-Rojo (1993); de Newnham ajustados por Contreras (1997) y Návar et al. (1997); de Kozak utilizados por Contreras (1997). Los modelos polinomiales incluyen por lo general hasta 5 parámetros estadísticos y adquieren formas extrañas en la base 0 parte distal del fuste. Los modelos de Newnham y Kozak incluyen también hasta 15 parámetros estadísticos.

El modelo de Biging predice el volumen comercial adecuadamente cuando se integra con la constante propuesta por Biging (1984) (Tabla 4). En contraste con otras funciones reportadas que requieren de otras técnicas, como análisis numéricos, para definir esta variable. La integración del modelo de Biging resultó en buenas estimaciones del volumen comercial para las especies estudiadas pero sólo para árboles con diámetros menores a $30 \mathrm{~cm}$
(Tabla 5). Para árboles mayores que $30 \mathrm{~cm}$ de diámetro el error, diferencia entre volumen rollo total estimado por la ecuación de Smalian y la integración del modelo de Biging, incrementó en forma de potencia con el diámetro para todas las especies estudiadas. Por esta razón, se recomienda estudiar la constante del modelo para ajustar esta tecnología a los volúmenes fustales de las especies estudiadas. A este respecto, Castillo (1988) realizó investigaciones de esta naturaleza en Pinus caribea de la Sabana, Oaxaca, México.

Los perfiles fustales de las cinco especies de la región de El Salto Durango, estimados por el modelo de Biging, presentan variación en el ahusamiento entre las categorías diamétricas. $P$. durangensis y $P$. cooperi forman curvas sigmoidales a partir de diámetros mayores que $15 \mathrm{~cm}$, mientras que el resto de las especies lo hace a partir de $20 \mathrm{~cm}$ de diámetro. Estas últimas forman curvas cuadráticas sencillas en diámetros menores que $20 \mathrm{~cm}$ (Fig. 2). Observaciones para la $1^{\mathrm{a}}$ y $2^{\mathrm{a}}$ derivada numérica de la función de Biging indicaron que el ahusamiento desde la base del fuste hasta cerca del punto de inflexión es similar para todas las especies.

El punto de inflexión de las especies es similar en altura entre las especies y va desde $4.5 \mathrm{~m}$ para diámetros de $30 \mathrm{~cm}$ hasta $9.0 \mathrm{~m}$ en diámetros de $60 \mathrm{~cm}$. Sin embargo, los puntos de inflexión en función de la altura total cambian desde $0.26 \mathrm{H}$ en $P$. durangensis, $0.28 \mathrm{H}$ en $P$. cooperi, hasta $0.30 \mathrm{H}$ en $P$. leiophylla, $P$. engelmannii y $P$. herrerae. Estos valores relativos son mayores que aquellos reportados por Demaerschalk y Kozak (1977) $(0.20 \mathrm{H}$ y $0.25 \mathrm{H}$ ) para algunas especies forestales en Columbia Britanica y por Newnham (1992) $(0.20 \mathrm{H})$ para cuatro especies forestales de Alberta. Pérez et al. (1990) reporta índices similares $(0.15 \mathrm{H}$ a $0.35 \mathrm{H})$ para Pinus oocarpa en Honduras. 

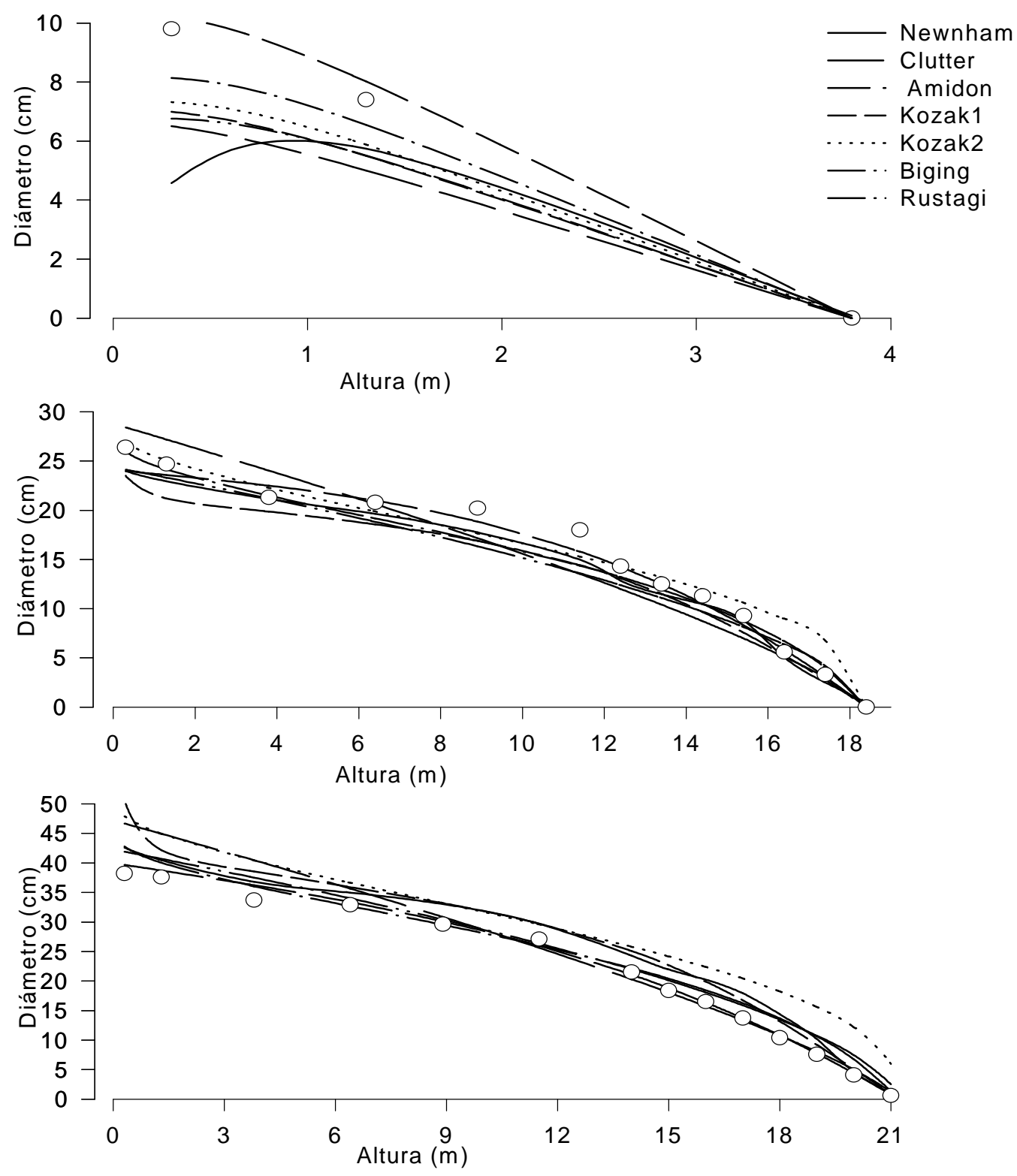

Figura 1. Ajuste de siete funciones de ahusamiento a los perfiles fustales sin corteza de $P$. durangensis de la región del Salto, Durango, México. 



Figura 2. Ajuste de la función de Biging a los perfiles fustales sin corteza de cinco especies de pino en la región del Salto, Durango, México. 
Tabla 4. Parámetros del ajuste del modelo de Biging (1984) para 5 pináceas de la región del Salto, Durango. México.

\begin{tabular}{||c|c|c||}
\hline \multicolumn{1}{|c|}{ ESPECIE } & B1 & B2 \\
\hline Pinus cooperi & 1.0518 & 0.2903 \\
Pinus durangensis & 1.0624 & 0.2941 \\
Pinus engelmannii & 1.0579 & 0.2894 \\
Pinus leiophylla & 1.0634 & 0.2939 \\
Pinus herrerae & 1.0424 & 0.2855 \\
\hline
\end{tabular}

A partir del punto de inflexión, $P$. durangensis y $P$. cooperison más cilíndricos y alcanzan el diámetro igual a cero a una altura mayor. Las otras especies alcanzan una altura de aproximadamente $2 \mathrm{~m}$ menor que $P$. durangensis y $P$. cooperi en la parte distal del fuste (Fig. 2).

El ahusamiento en la parte distal del fuste es mas pronunciado en $P$. leiophylla, $P$. engelmannii y $P$. herrerae. Sin embargo, estas especies poseen una longitud fustal cónica similar a $P$. durangensis y $P$. cooperi. A este respecto, Larson (1963) demostró que las diferencias en el perfil diamétrico se deben parcialmente al tamaño de la copa viva que se distribuye en el fuste y el tamaño de las ramas. Factores tales como el sitio y la densidad influencian el ahusamiento a través de su efecto en el desarrollo de la copa.

\section{CONCLUSIONES}

El ajuste y validación de 7 funciones de ahusamiento mostró que el modelo de Biging describió mejor los perfiles diamétricos sin corteza de 5 especies comerciales de pino de los bosques de la región del Salto, Durango. Los perfiles diamétricos estimados por el modelo mostraron que existen diferencias en el ahusamiento entre las especies observándose que $P$. durangensis y $P$. cooperi son más cilíndricos que $P$. engelmannii, $P$. leiophylla y $P$. herrerae. Se recomienda el uso del modelo de Biging para estimar los perfiles fustales $y$ volúmenes comerciales de árboles menores a $30 \mathrm{~cm}$ de diámetro normal de las pináceas de la región del Salto, Durango.

\section{RECONOCIMIENTO}

Los autores de este reporte de investigación desean hacer patente su agradecimiento al Consejo Nacional de Ciencia y Tecnología por los apoyos otorgados a través del proyecto de investigación 28536-B. El Ing. Miguel A. Romero Sarmiento es también reconocido por su cooperación para el desarrollo de este trabajo.

\section{REFERENCIAS}

Amidon, E. L. 1984. A general taper functional form to predic bole volume for five mixted conifer especies in California. For. Sci. 30:166-171.

Biging, G.S. 1984. A compatible volume taper fuction for Alberta trees. For. Sci. 30:1103-1117.

Castillo-Santiago, M.A. 1988. Modelo para estimación de incremento y producción maderable neta en Pinus caribea var hondurensis Barr. Y Golf., de la Sabana, Oaxaca. Tesis profesional. Chapingo, México. 81 p. 
Tabla 5. Mapa de distribución de la muestra para ajustar y validar 7 modelos de ahusamiento a 5 especies de coníferas del Salto, Durango, México

\begin{tabular}{|c|c|c|c|c|c|c|c|c|c|c|c|}
\hline \multirow[b]{2}{*}{$\mathrm{H}$} & \multirow[b]{2}{*}{$E_{i}$} & \multicolumn{9}{|c|}{ CATEGORIA DE DIAMETRO } & \multirow[b]{2}{*}{ Total } \\
\hline & & 10 & 15 & 20 & 25 & 30 & 35 & 40 & 45 & $>=50$ & \\
\hline \multirow[t]{5}{*}{5} & 1 & $38(13)$ & $17(6)$ & $2(1)$ & & & & & & & $57(20)$ \\
\hline & 2 & $35(18)$ & $21(11)$ & $3(1)$ & & & & & & & $59(30)$ \\
\hline & 3 & $42(14)$ & $22(7)$ & 3 & & & & & & & $67(21)$ \\
\hline & 4 & $50(13)$ & $27(5)$ & 9 & & & & & & & $86(18)$ \\
\hline & 5 & $30(10)$ & 12(2) & $4(1)$ & & & & & & & $46(13)$ \\
\hline \multirow[t]{5}{*}{10} & 1 & $9(7)$ & $21(11)$ & $32(6)$ & $9(3)$ & $6(1)$ & $5(1)$ & & & & $82(29)$ \\
\hline & 2 & $6(3)$ & $31(11)$ & $36(12)$ & $16(4)$ & 2 & 5 & & & & $96(30)$ \\
\hline & 3 & $15(6)$ & $34(23)$ & $40(13)$ & $19(3)$ & 1(3) & & & & & 109(48) \\
\hline & 4 & $6(4)$ & $37(13)$ & $36(15)$ & $10(3)$ & 2 & & & & & $91(35)$ \\
\hline & 5 & $10(3)$ & $43(6)$ & $31(9)$ & $10(4)$ & & & & & & $94(22)$ \\
\hline \multirow[t]{5}{*}{15} & 1 & & $3(2)$ & $19(11)$ & $41(14)$ & $30(4)$ & $17(3)$ & $5(1)$ & & & $115(35)$ \\
\hline & 2 & & $5(6)$ & $18(12)$ & $41(7)$ & $23(4)$ & 6 & & & & 93(29) \\
\hline & 3 & & $3(2)$ & $32(14)$ & $29(9)$ & $19(2)$ & $12(1)$ & 2 & & & $97(28)$ \\
\hline & 4 & & 1(4) & $21(7)$ & $23(12)$ & $21(5)$ & $11(2)$ & & & & $77(30)$ \\
\hline & 5 & & $4(2)$ & $13(5)$ & $23(7)$ & $15(7)$ & $3(3)$ & 1 & & & $59(24)$ \\
\hline \multirow[t]{5}{*}{20} & 1 & & & & $8(2)$ & $20(11)$ & $28(11)$ & $15(4)$ & $5(2)$ & 1 & $77(30)$ \\
\hline & 2 & & & $3(1)$ & $3(5)$ & $23(6)$ & $18(2)$ & $5(2)$ & $3(1)$ & & $55(17)$ \\
\hline & 3 & & & 1 & 1(1) & $9(2)$ & $11(1)$ & $4(3)$ & $0(1)$ & & $26(8)$ \\
\hline & 4 & & & $1(1)$ & $3(5)$ & $3(5)$ & $13(3)$ & $11(1)$ & 2 & 2 & $35(15)$ \\
\hline & 5 & & & & $4(1)$ & $9(8)$ & $8(3)$ & $8(1)$ & 3 & & $32(13)$ \\
\hline \multirow[t]{5}{*}{25} & 1 & & & & & $2(2)$ & $7(6)$ & $24(1)$ & 6 & 4 & $43(9)$ \\
\hline & 2 & & & & $0(1)$ & $2(1)$ & $5(1)$ & $13(2)$ & 8 & 2 & $30(5)$ \\
\hline & 3 & & & & & 1 & 2 & $4(1)$ & 5 & 3 & $15(1)$ \\
\hline & 4 & & & & & 1 & $1(2)$ & 2 & 4 & 3 & $11(2)$ \\
\hline & 5 & & & & & & $3(3)$ & 1(4) & $2(1)$ & 3 & $9(9)$ \\
\hline \multirow[t]{5}{*}{30} & 1 & & & & & & & $0(1)$ & $1(1)$ & 3 & $4(2)$ \\
\hline & 2 & & & & & & & 1 & $1(1)$ & $2(1)$ & $4(2)$ \\
\hline & 3 & & & & & & & 2 & 2 & & $4(0)$ \\
\hline & 4 & & & & & & & & & & $0(0)$ \\
\hline & 5 & & & & & & & & & 1(1) & 1(1) \\
\hline \multirow[t]{5}{*}{35} & 1 & & & & & & & & & $2(1)$ & $2(1)$ \\
\hline & 2 & & & & & & & & & 3 & $3(0)$ \\
\hline & 3 & & & & & & & & & & $0(0)$ \\
\hline & 4 & & & & & & & & & & $0(0)$ \\
\hline & 5 & & & & & & & & & & $0(0)$ \\
\hline \multirow[t]{2}{*}{ Total } & & 241 & 281 & 304 & 240 & 189 & 155 & 98 & 43 & 29 & 1579 \\
\hline & & (91) & $(111)$ & $(109)$ & (81) & (61) & (43) & $(21)$ & (7) & (3) & (527) \\
\hline
\end{tabular}

NOTA: $\mathrm{H}=$ altura, $\mathrm{E}=$ Especie $(\mathrm{i}=1=P$. cooperi, $2=P$. durangensis, $3=P$. engelmannii, $4=P$. leiophylla y $5=P$. herrerae); 38 (13)=Número de árboles para el ajuste (Número de árboles para validar). 
Clutter, J. L. 1980. Development of taper functions from variable-top merchantable volume equations. For. Sci. 26: 117-120.

Contreras-Aviña, J.C. 1997. Ecuaciones de volumen y ahusamiento para Pinus durangensis Mart. y Pinus teocote Sch. et Cham. Del ejido Vencedores, San Dimas, Durango. Tesis de Maestría. Facultad de Ciencias Forestales. U.A.N.L. Linares, Nuevo León. 78 p.

Demaerschlk, J.P, and Kozak, A. 1977. The whole - bole system: a conditioned dual- equation system for red and loblolly pine for precise prediction of tree profiles. Can. J. For. Res. 7:488497.

Kozak, A. 1988. A variable-exponent taper equation. Can. J. For. Res. 18:13631368.

Larson, P. R. 1963. Stem form development of forest trees. For. Sci Monograf. 5.

Návar J., P. A. Dominguez-Calleros, J. C. Contreras-Aviña y C. Estrada M. 1997. Ajuste de siete modelos de ahusamiento a los perfiles fustales de Pinus hartwegii Lindl. Del Noreste de México. Agrociencia 31: 73-81.

Newnham, R.M. 1992. Variable-form taper functions for four Alberta tree species. Can. J. For. Res. 22: 210-223.
Pérez, D.N., Burkhart, H.E., and Stiff, C.T. 1990. A variable-form taper fuction for Pinus occarpa Schiede in central Honduras. For. Sci. 36:186-191.

Rentería-Anima, J. B., H. RamírezMaldonado y F. J. Zamudio-Sánchez. 1995. Estimación de volumen comercial de Pinus cooperi blanco mediante modelos de ahusamiento en Durango. II Congreso de Mexicano sobre Recursos Forestales. Resumen de ponencias. Desarrollo sustentable redefiniendo el papel de los recursos forestales. Montecillo, México. 91 p.

Rustagi, K. P. y R. S. Loveless, 1991. Compatible variable-form volume and stem profile equations for Douglas-fir. Can. J. For. Res. 21:143-151.

Torres-Rojo, J. M., S. O. Magaña T. y G. A. Valles G. 1993. Funciones de ahusamiento para 8 especies Forestales del Estado de México. I Congreso Mexicano de Recursos Forestales, Resumen de Ponencias. Saltillo, Coahuila. 109 p.

Zepeda-Bautista, M. y A. DominguezPereda. 1997. Ecuaciones de ahusamiento para tres especies de pino, del ejido EI Largo, Chihuahua. III Congreso Mexicano sobre Recursos Forestales. Resumen de ponencias. Nuevos paradigmas. Linares, Nuevo León. $122 \mathrm{p}$. 
